\title{
Cumulative incidence of $A C L$ reconstruction after $A C L$ injury in adults: Role of age, sex and race
}

\author{
Jamie E. Collins ${ }^{1,3}$, Jeffrey N. Katz, MD, MSc ${ }^{1,2}$, Laurel A. Donnell-Fink, MPH ${ }^{1}$, Scott D. \\ Martin, $\mathbf{M D}^{1}$, and Elena Losina, $\mathbf{P h D}^{1,3}$ \\ ${ }^{1}$ Department of Orthopedic Surgery, Brigham and Women's Hospital, Harvard Medical School, \\ Boston, MA \\ ${ }^{2}$ Division of Rheumatology, Immunology and Allergy, Brigham and Women's Hospital, Harvard \\ Medical School, Boston, MA \\ ${ }^{3}$ Department of Biostatistics, Boston University School of Public Health, Boston, MA
}

\begin{abstract}
Background-Anterior cruciate ligament (ACL) injuries are common, potentially disabling and frequently prompt surgical reconstruction. The utilization of ACL reconstruction among ACLinjured individuals has not been examined rigorously.

Purpose-This study reports the 3-year cumulative incidence of ACL reconstruction among adults with ACL injury and compares demographic and clinical characteristics of ACL-injured patients that do and do not go on to have ACL reconstruction.
\end{abstract}

Study Design-Retrospective cohort study

Methods-We used a tertiary healthcare system patient data repository to identify patients diagnosed with an ACL injury between January 1, 2001 and December 31, 2007. We obtained follow-up data to determine how many patients with ACL injury underwent ACL reconstruction within three years of ACL injury diagnosis. We used stratified analyses to examine incidence rates separately by sex, age, race, primary language, socioeconomic status, and health insurance status. We built multivariable logistic regression models to examine the association of patient characteristics with utilization of ACL reconstruction.

Results-There were 2,304 patients diagnosed with an ACL injury between 2001 and 2007 with a mean age of 47. The 3-year cumulative incidence of ACL reconstruction after ACL injury diagnosis was $22.6 \%$ (95\% CI: $(20.9 \%-24.3 \%)$ ). Eighty-six percent of patients undergoing reconstruction did so within 6 months of injury diagnosis while $94 \%$ underwent reconstruction within 1 year. In multivariable models several patient features were independently associated with a higher adjusted odds of undergoing ACL reconstruction, including male sex (adjusted odds ratio (aOR) 1.4, 95\% CI: $(1.1-1.7)$ ), younger age (aOR per decade 1.8, 95\% CI: $(1.7-2.0)$ ), white race (aOR 1.4, 95\% CI: $(0.94-1.9)$ ), higher socioeconomic status (aOR 1.4, 95\% CI: (1.04-1.8) for high vs. low SES; aOR 1.3, 95\% CI: $(1.02-1.8)$ for medium vs. low SES), and private health insurance (aOR 1.9, 95\% CI: $(1.04-3.5)$ ) vs. self-pay.

Conclusions-Less than a quarter of patients diagnosed with ACL injury underwent ACL reconstruction over three years from diagnosis. The odds of having surgery were higher for males,

Correspondence: Jamie E Collins, Orthopedics and Arthritis Center for Outcomes Research, Department of Orthopedic Surgery, Brigham and Women's Hospital, 75 Francis Street, BC 4-016, Boston, MA, 02115, JCOLLINS13@PARTNERS.ORG.

At which institution the study was performed: Brigham and Women's Hospital, Boston MA 
whites, younger patients, patients with higher socioeconomic status, and patients with private health insurance.

\section{Keywords}

adult; anterior cruciate ligament (ACL); injury; reconstruction

\section{Introduction}

Anterior cruciate ligament (ACL) injuries are a frequent source of disability and health resource utilization. Incidence rates for ACL injury are difficult to assess, since not all individuals with ACL injury seek medical care. Estimates reach up to 250,000 injuries per year ${ }^{7}$.

ACL reconstruction has been shown to be a successful and cost effective treatment for ACL injury $5,6,11$. According to national data, there were approximately 125,000 ACL reconstructions performed in United States in $2006^{9}$. While the number of persons undergoing ACL reconstruction appears to be increasing annually ${ }^{3,9,12}$, the proportion of ACL-injured individuals who undergo ACL reconstruction has not been examined rigorously.

Our study objectives were to determine the frequency of ACL reconstruction among those with ACL injury diagnosis and to identify patient characteristics associated with greater likelihood of ACL reconstruction. We sought to identify subgroups less likely to utilize ACL reconstruction.

\section{Methods}

Setting

The study took place at an urban tertiary academic medical center. Approximately 98,000 patients are cared for by primary care practices annually at affiliated health care centers, at both community and hospital-based clinics. Approximately 59,000 patients are seen annually in the emergency department. The department of orthopedic surgery consists of more than forty physicians at four locations. The hospital is a 780-bed adult medical center located in a racially and socioeconomically diverse urban neighborhood. In addition to the main hospital the center has a distributed campus that includes a 120-bed community hospital and several neighborhood health centers located throughout the city. There is also a large satellite center in a more suburban area. The hospital is part of an integrated healthcare system. Our sample consists of adults only since our medical center does not care for the pediatric population. The study was approved by the institution's Human Research Committee.

\section{Data Source}

Data were obtained from the institution's Research Patient Data Repository (RPDR), a clinical data registry that gathers medical record data from the healthcare system. The RPDR contains information on patient demographics, diagnoses, procedures, and inpatient and outpatient encounters. An online query tool allows users to create complex queries, and with proper IRB approval - to obtain detailed de-identified patient-level data.

\section{Sample}

We queried the RPDR for all patients diagnosed with an ACL injury (ICD-9 844.2, sprain of cruciate ligament of knee) at the institution between January 1, 2001 and December 31, 
2007. We requested detailed patient data for this cohort on diagnoses and procedures through January 1, 2011, ensuring that each patient had 3 years of follow-up. If a subject had more than one diagnosed ACL injury, we selected the earliest diagnosis date. We included patients with any diagnosis of ACL injury, and as a sensitivity analysis included only those patients for whom ACL injury was the principle diagnosis.

\section{Defining primary outcome}

ACL reconstruction was determined by searching the procedures database for CPT 29888 (Arthroscopically aided anterior cruciate ligament repair/augmentation or reconstruction). Patients with ACL reconstruction prior to ACL injury diagnosis and patients with more than one ACL reconstruction were excluded from the analysis. Time to ACL reconstruction was calculated as time from first documentation of ACL injury at the institution to time of ACL reconstruction.

As a secondary analysis we also included patients with ICD-9 81.45 (Other repair of the cruciate ligaments) and CPT 27409 (Repair, primary, torn ligament and/or capsule, knee; collateral and cruciate ligaments). These codes are usually reserved for complex ligamentous injuries, but can be used for ACL reconstruction.

\section{Demographic and Clinical Variables}

Demographic data (sex, age, race/ethnicity, primary language, health insurance) were provided by the RPDR. Patients were grouped into six age strata: 20-29, 30-39, 40-49, 50-59, 60-69 and 70+. Race was categorized as white, non-white, and unknown. Primary language was categorized as English, Spanish, and other. Primary health insurance was grouped into 5 categories: private insurance, Medicare, free care (e.g., Welfare, Medicaid), self-pay, and other. We obtained data from the 2000 US Census $^{2}$ and used the zip code of each patient's residence, provided by the RPDR, to obtain ecological information on socioeconomic status (SES). For each zip code we assessed percent non-white, percent foreign born, percent not completing high school, percent below poverty, and median income. For each of these socio-demographic indicators, we first created 4-level categorical variables based on quartiles. To account for multidimensionality of socio-demographic status we created an index of socio-demographic deprivation by summing the 5 indicators. We divided the risk score into thirds based on tertiles to classify patients into low, medium, or high SES neighborhoods ${ }^{10}$.

\section{Validation}

We reviewed the electronic medical records of 100 randomly selected patients to validate the diagnoses and procedures reported in RPDR. Out of the 100 randomly selected patients, 83 ACL injuries (83.0\%, 95\% CI: $(74.2 \%-89.8 \%))$ and all 25 ACL surgical reconstructions $(100 \%, 95 \%$ CI: $86.3 \%-100 \%))$ were confirmed. Electronic medical record review identified an additional 4 ACL surgeries which were not reported in RPDR, suggesting a sensitivity of ACL surgical reconstruction of 25/29 (86.2\%, 95\% CI: (68.3\% - 96.1\%)). Of the 83 patients with ACL injury confirmed, $87 \%$ had this diagnosis made by an orthopedic surgeon.

The BWH Department of Orthopedic Surgery provided us a list of all ACL reconstructions performed between 2007 and 2010. We compared the department list of ACL reconstructions to the data provided by RPDR. RPDR was inclusive of all 71 patients who had ACL reconstruction surgery between 2007 and 2010 as documented by the department. 


\section{Statistical Analysis}

We defined the cumulative incidence of ACL reconstruction as the proportion of ACLinjured patients who underwent ACL reconstruction within three years of the initial documentation of ACL injury. In addition to deriving the overall ACL reconstruction incidence rates, we present stratified rates by sex, age, race, primary language, SES, and health insurance status.

We built multivariable logistic regression models to identify independent predictors of ACL reconstruction among those with ACL injury. All covariates were statistically significantly related to ACL reconstruction in bivariate analysis, therefore we included all covariates in the initial multivariable model. We then used likelihood ratio statistics and odds ratios to determine the final model. We hypothesized that the association between ACL reconstruction and sex may depend on race and age and the association between ACL reconstruction and race may depend on age. We tested these hypotheses by including interaction terms in our multivariable logistic models. Kaplan-Meier survival curves were used to estimate the cumulate rate of ACL reconstruction over 3 years after injury diagnosis and to compare the unadjusted rates of ACL reconstruction by sex. All analyses were conducted using SAS software version 9.2 (SAS Institute, Cary, NC).

\section{Results}

Sample

The RPDR query returned 2,370 patients diagnosed with an ACL injury at the institution between 2001 and 2007. Of these, 48 had a previous ACL reconstruction and an additional 18 had more than one reconstruction. Since laterality could not be established from the RPDR, these patients were removed from the analysis, leaving 2,304 patients. The cohort was $47 \%$ female and 53\% male and the average age was 47.1 (SD 13.9) (Table 1). Ten percent of the cohort was 20-29 at the time of ACL injury, 22\% was 30-39, 27\% was 40-49, $21 \%$ was $50-59,13 \%$ was $60-69$ and $6.5 \%$ was 70 or older. Sixty-four percent of the cohort was white, $17 \%$ non-white, and race was unknown for $19 \%$ of the cohort. Ninety-four percent of the cohort listed English as primary language, 3\% listed Spanish, and 2\% listed another language. The majority of patients had private health insurance (75\%), followed by free care (9\%), Medicare (8\%), self-pay (4\%) and other (4\%).

\section{Overall 3-year cumulative incidence of $A C L$ reconstruction}

520 patients underwent ACL reconstruction at the institution within 3 years of ACL injury diagnosis, for a 3 -year cumulative incidence of $22.6 \%$ (95\% CI: $(20.9 \%-24.3 \%)$ ). Eightysix percent of patients undergoing reconstruction did so within 6 months of ACL injury diagnosis while $94 \%$ underwent reconstruction within 1 year, for a 1-year cumulative incidence of $21.3 \%$ (95\% CI: $19.6 \%-22.9 \%)$ ).

\section{Bivariate associations between patient features and $A C L$ reconstruction}

Overall, males were more likely than females to undergo ACL reconstruction following ACL injury diagnosis (26\% vs $19 \%, \mathrm{p}<0.001)$ (Table 2). The likelihood of reconstruction among ACL-injured patients decreased with each decade of age: for example 45\% of 20-29 year olds underwent reconstruction compared to $8 \%$ of $60-69$ years olds $(\mathrm{p}<0.001)$. Whites were more likely to utilize reconstruction than non-whites (23\% vs $16 \%$ ), while $26 \%$ of patients with unknown race underwent reconstruction $(\mathrm{p}=0.002)$.

Patients with English as their primary language were the most likely to undergo reconstruction (23\%), followed by patients speaking a language other than English or Spanish (16\%) while Spanish speaking patients were the least likely to undergo 
reconstruction (8\%, $\mathrm{p}=0.003)$. Since English speakers had a comparable risk of ACL reconstruction compared to patients speaking a language other than English or Spanish we collapsed the English and other language groups into one group and focused our comparison on Spanish vs non-Spanish speakers in the multivariable models. Patients in high and medium SES neighborhoods had a similar likelihood of reconstruction, while those in low SES neighborhoods were the least likely to undergo reconstruction, though this difference was not statistically significant (24\% high SES, $24 \%$ medium SES, $20 \%$ low SES, p=0.06). Patients with private $(26.7 \%)$ and other $(27.6 \%)$ insurance were the most likely to undergo reconstruction, followed by those self-paying (16.9\%) and with free care (14.5\%). Patients using Medicare as the primary form of health insurance were the least likely to undergo reconstruction $(7.7 \%)(\mathrm{p}<0.001)$.

\section{Stratified analysis \& multivariable models}

Zip code based SES level, language, race, and insurance status were highly associated with each other (Chi-square p-value $<0.001$ ); thus, we used likelihood ratio statistics and odds ratios to determine the relative importance of each predictor in order to create a more parsimonious model. After adjustment language was dropped from the model. After dropping language from the model all remaining predictors were statistically significant $(\mathrm{p}<0.05)$. This model had the optimal likelihood ratio statistic and was the model chosen by forward, backward, and stepwise selection. The final model included sex, race, SES, insurance status, and age group (as a continuous variable). Males had 1.4 fold greater adjusted odds of reconstruction compared to females (95\% CI: $(1.1-1.7)$ ); whites had 1.4 times the adjusted odds of reconstruction compared to non-whites (95\% CI: $(0.95-1.9))$ and a similar odds of reconstruction compared to those with unknown race (aOR: $0.81,95 \%$ CI: $(0.62-1.1))$. There was a linear trend with age group: each decrease in decade of age was associated with 1.8 times the adjusted odds of reconstruction (95\% CI: $(1.7-2.0)$ ). Patients in the high (aOR: 1.4, 95\% CI: $(1.04-1.8)$ ) and medium (aOR: 1.3, 95\% CI: $(1.02$ -1.8)) SES groups had higher adjusted odds of reconstruction compared to patients in the lowest SES group. Finally, compared to patients self-paying, patients with private insurance had 1.9 fold greater adjusted odds of reconstruction (95\% CI: $(1.04-3.5)$ ); patients with other insurance had 1.7 fold greater adjusted odds (95\% CI: $(0.8-3.8))$; and patients with Medicare had 1.6 fold greater adjusted odds (95\% CI: $(0.66-3.7)$ ). The odds of undergoing reconstruction were similar for patients with free care compared to self-pay (aOR: 1.1, 95\% CI: $(0.53-2.3))$.

Stratified analyses (Figure 1) show that among patients younger than 29, ACL-injured females are more likely to undergo reconstruction than ACL-injured males, whereas among ACL-injured patients 30-60 years old, males are more likely to undergo surgery. However these differences in sex effect across age groups were not statistically significant in a formal test for interaction. The trend for whites to be more likely to undergo reconstruction than non-whites held across all age groups. Among non-whites, females were much less likely than males to undergo reconstruction (10.6\% vs. $21.4 \%)$ while among whites the difference was less pronounced, with $19.8 \%$ of females undergoing reconstruction vs $26.2 \%$ of males. The formal test for interaction was not statistically significant $(\mathrm{p}=0.20)$.

The Kaplan-Meier curves show that the probability of reconstruction is higher for males than for females at all time points (logrank p-value < 0.001) (Figure 2).

\section{Sensitivity Analyses}

We repeated the analysis including only patients whose principle diagnosis was ACL tear (ICD-9 81.45). Of the 2,304 patients identified in our sample, 1,703 (73.9\%) had ACL tear as the principle diagnosis. The remaining 601 patients had ACL tear as a secondary 
diagnosis. Other principle diagnoses included tear of medial cartilage or meniscus (ICD-9 83.60; $\mathrm{n}=176$ ), pain in joint involving lower leg (ICD-9 719.46; $\mathrm{n}=94$ ), and tear of lateral cartilage or meniscus (ICD-9 83.61; $n=81$ ). 455 of the 1,703 patients underwent ACL reconstruction at the institution within 3 years of ACL injury diagnosis, for a 3-year cumulative incidence of $26.7 \%$ (95\% CI: $(24.6 \%-28.9 \%)$ ).

We repeated the analysis again including the additional procedure codes (ICD-9 81.45 and CPT 27409) that may be used for complex ligament injuries, including ACL tear. This identified 2,262 patients with ACL injury that met our inclusion criteria. Of these, 618 underwent ACL reconstruction at the institution within 3 years of ACL injury for a 3-year cumulative incidence of $27.3 \%$ (95\% CI: $(25.5 \%-29.2 \%)$ ). While the codes 81.45 and 27409 were more likely to be used in older than patients, the associations between potential covariates and reconstruction were the same as reported above.

\section{Discussion}

We used a patient data repository available to researchers to identify patients diagnosed with an ACL injury and evaluated the rate of ACL reconstruction among these ACL-injured patients. The 3-year cumulative incidence of ACL reconstruction among ACL-injured patients was $22.6 \%$ (95\% CI: $(20.9 \%-24.3 \%))$. We found that ACL reconstruction rates varied by age, sex, race, socioeconomic status, and insurance status: males were more likely to have an ACL injury reconstructed than females, whites were more likely that non-whites, younger people were more likely to undergo ACL reconstruction, patients of higher socioeconomic status were more likely to undergo ACL reconstruction, and patients with private insurance were more likely to undergo ACL reconstruction.

Our study provides age, sex, and race stratified risks of ACL reconstruction among patients with ACL injury, potentially permitting calculation of injury rates from data on surgical rates. In fact, the National Survey of Ambulatory Surgery (NSAS) provides a comprehensive overview of ambulatory surgical procedures performed in the US, including ACL reconstruction ${ }^{1,9}$. Thus, our data could be combined with NSAS data to estimate ACL injury rates from ACL reconstruction rates.

Few studies have examined the rate of ACL reconstruction among those with ACL injury. One study reported a cumulative incidence of ACL reconstruction within 6 weeks of index hospitalization of $58 \%{ }^{4}$. The study found unadjusted differences in reconstruction rates by age group and by race, but not by sex. Since the goal of the study was to evaluate rates of reinjury, adjusted and stratified analyses for rates of ACL reconstruction were not provided. In addition, the cohort was comprised of active-duty US Army personnel and was predominately male $(91 \%)$ and young (90\% under 40$)$. All subjects had full access to health care, limiting the generalizability. Many other studies have evaluated the risk of ACL injury by demographic variables, though most sample relatively young, healthy populations $3,8,13,14$. For example, a study of students at West Point found little difference in the individual risk of an ACL tear between males and females during their 4-year college career ${ }^{14}$.

A recent national community based study in Sweden found surgical rates similar to the rates presented in our study. In the Swedish study $47.7 \%$ of injured 21-30 year olds underwent reconstruction, compared to $45.3 \%$ in our cohort ${ }^{15}$. The proportion undergoing reconstruction decreased with each decade of age in a similar manner: the reconstruction rate was $34.3 \%$ of $31-40$ year olds, $21.4 \%$ for $41-50$ year olds, and $6.6 \%$ for those $50+$. The overall surgical rate was higher, $36.4 \%$ compared to $22.6 \%$ in our cohort, although this study included patients under 20 years old therefore a higher overall rate is to be expected. 
We found that only $45 \%$ of 20-29 year old patients with ACL injury underwent reconstruction within 3 years of initial diagnosis. This may be an underestimate, since subjects diagnosed with an injury at the institution could undergo ACL reconstruction elsewhere, however it is remarkably close to the estimate from the Swedish cohort presented above $^{15}$. In addition, we found that sex, race, socioeconomic status, and health insurance status affect the rate of ACL reconstruction. These effects persisted in adjusted analysis, suggesting that access to care could be an obstacle for patients of lower socioeconomic status or without health insurance.

Results of this study should be interpreted in the context of the study design. As noted, the RPDR only identified ACL reconstructions at the institution; therefore, subjects diagnosed at the institution and undergoing surgery at a different institution were misclassified as not having ACL reconstruction. Because the study was done in a major referral center, losses to other centers are likely uncommon; they would result in underestimation of the rates of surgery. Also, the RPDR query did not provide information on the severity of the ACL injury or whether the injury was isolated or a complex ligamentous injury. These may be important factors in the decision to undergo surgery. In addition, the RPDR did not provide information on laterality. Patients may have had ACL tear reported in one knee and ACL reconstruction in the other knee. We excluded patients with ACL reconstruction prior to ACL tear and patients with more than one ACL reconstruction. As a sensitivity analysis we repeated the analysis including these 66 patients, and the results were similar to those of the parent analysis. Because this was not a population-based study we were not able to directly estimate the rate of ACL injury.

Our sample consists of adults only since our medical center does not care for the pediatric population. Thus, these data cannot be extrapolated to the younger $(<20$ year old) population and the overall reconstruction rate of $22.6 \%$ is generalizable only to a population with a similar age distribution. Much of the literature on ACL injury and reconstruction focuses on the younger population yet the majority of ACL injured patients are indeed adults; thus, our study fills an important niche by focusing on the adult population. A recent national community based study performed in Sweden found similar age-stratified surgical rates to ours, supporting the validity of our findings. We re-weighted the rates provided by the Swedish cohort using the age distribution of our population and found the age-weighted rate to be $20.8 \%$ in the Swedish cohort, which is comparable to our finding of $22.6 \%$.

Based on the results of our validation study and sensitivity analysis we may be somewhat underestimating our overall rate of surgical conversion. Using the results of the validation exercise, we conclude that by adjusting for potential underestimation by $28.4 \%$, the corrected incidence rate would be $31.5 \%$. We acknowledge that this is a limitation in our study; however, any study utilizing an administrative database is likely to suffer from the same limitations. In addition, our findings were remarkably close to those from Nordenvall et al. Given the data available to us we present the most accurate 3-year cumulative incidence of ACL reconstruction to date and we urge further research in the area.

To our knowledge this is the first study to report the 3-year cumulative incidence rates of ACL reconstruction among those diagnosed with an ACL injury. In this study $22.6 \%$ of patients with ACL injury underwent reconstruction within 3 years of injury diagnosis. Patient characteristics were associated with utilization of ACL reconstruction after ACL injury: the odds of having an injury reconstructed surgically were lower for females, nonwhites, patients in lower socioeconomic status neighborhoods, patients with free care or no health insurance, and older patients, even after adjusting for other covariates. Future studies are needed to better understand why certain patients are less likely to utilize ACL 
reconstruction and to ensure that all patients are given all the information necessary to make an informed decision about surgery.

\section{Acknowledgments}

Support:

Grants from NIH/NIAMS including T32AR055885 Training in Orthopaedic and Musculoskeletal Clinical Research, K24 AR 057827 and P60 AR 047782; and from Harvard Catalyst | The Harvard Clinical and Translational Science Center (NIH Award UL1 RR 025758 and financial contributions from Harvard University and its affiliated academic health care centers). The content is solely the responsibility of the authors and does not necessarily represent the official views of Harvard Catalyst, Harvard University and its affiliated academic health care centers, the National Center for Research Resources, or the National Institutes of Health.

\section{References}

1. National Center for Health Statistics. National Survey of Ambulatory Surgery. [Accessed October 15, 2011] Available at: http://www.cdc.gov/nchs/nsas.htm

2. U.S. Census Bureau American FactFinder. [Accessed October 4, 2011] 2011. Available at: factfinder.census.gov

3. Csintalan RP, Inacio MC, Funahashi TT. Incidence rate of anterior cruciate ligament reconstructions. Perm J. 2008; 12(3):17-21. [PubMed: 21331205]

4. Dunn WR, Lyman S, Lincoln AE, Amoroso PJ, Wickiewicz T, Marx RG. The effect of anterior cruciate ligament reconstruction on the risk of knee reinjury. Am J Sports Med. 2004; 32(8):19061914. [PubMed: 15572320]

5. Farshad M, Gerber C, Meyer DC, Schwab A, Blank PR, Szucs T. Reconstruction versus conservative treatment after rupture of the anterior cruciate ligament: cost effectiveness analysis. BMC Health Serv Res. 11:317. [PubMed: 22098703]

6. Gottlob CA, Baker CL Jr. Pellissier JM, Colvin L. Cost effectiveness of anterior cruciate ligament reconstruction in young adults. Clin Orthop Relat Res. 1999; (367):272-282. [PubMed: 10546625]

7. Griffin LY, Albohm MJ, Arendt EA, et al. Understanding and preventing noncontact anterior cruciate ligament injuries: a review of the Hunt Valley II meeting, January 2005. Am J Sports Med. 2006; 34(9):1512-1532. [PubMed: 16905673]

8. Janssen KW, Orchard JW, Driscoll TR, van Mechelen W. High incidence and costs for anterior cruciate ligament reconstructions performed in Australia from 2003-2004 to 2007-2008: time for an anterior cruciate ligament register by Scandinavian model? Scand J Med Sci Sports.

9. Kim S, Bosque J, Meehan JP, Jamali A, Marder R. Increase in outpatient knee arthroscopy in the United States: a comparison of National Surveys of Ambulatory Surgery, 1996 and 2006. J Bone Joint Surg Am. 2011; 93(11):994-1000. [PubMed: 21531866]

10. Losina E, Wright EA, Kessler CL, et al. Neighborhoods matter: use of hospitals with worse outcomes following total knee replacement by patients from vulnerable populations. Arch Intern Med. 2007; 167(2):182-187. [PubMed: 17242320]

11. Lubowitz JH, Appleby D. Cost-effectiveness analysis of the most common orthopaedic surgery procedures: knee arthroscopy and knee anterior cruciate ligament reconstruction. Arthroscopy. 27(10):1317-1322. [PubMed: 21855268]

12. Lyman S, Koulouvaris P, Sherman S, Do H, Mandl LA, Marx RG. Epidemiology of anterior cruciate ligament reconstruction: trends, readmissions, and subsequent knee surgery. J Bone Joint Surg Am. 2009; 91(10):2321-2328. [PubMed: 19797565]

13. Mihata LC, Beutler AI, Boden BP. Comparing the incidence of anterior cruciate ligament injury in collegiate lacrosse, soccer, and basketball players: implications for anterior cruciate ligament mechanism and prevention. Am J Sports Med. 2006; 34(6):899-904. [PubMed: 16567461]

14. Mountcastle SB, Posner M, Kragh JF Jr. Taylor DC. Gender differences in anterior cruciate ligament injury vary with activity: epidemiology of anterior cruciate ligament injuries in a young, athletic population. Am J Sports Med. 2007; 35(10):1635-1642. [PubMed: 17519438] 
15. Nordenvall R, Bahmanyar S, Adami J, Stenros C, Wredmark T, Felländer-Tsai L. A PopulationBased Nationwide Study of Cruciate Ligament Injury in Sweden, 2001-2009 Incidence,

Treatment, and Sex Differences. The American Journal of Sports Medicine. 2012; 40(8):18081813. [PubMed: 22684536] 
What is known about the subject: ACL injuries are relatively common, with estimates ranging up to 250,000 per year. ACL reconstruction has been shown to be a successful treatment for ACL injury, and the number of ACL reconstructions performed in the United States appears to be increasing annually.

What this study adds to existing knowledge: To our knowledge this is the first study to report the 3-year cumulative incidence rates of ACL reconstruction among those diagnosed with an ACL injury in a US cohort. 


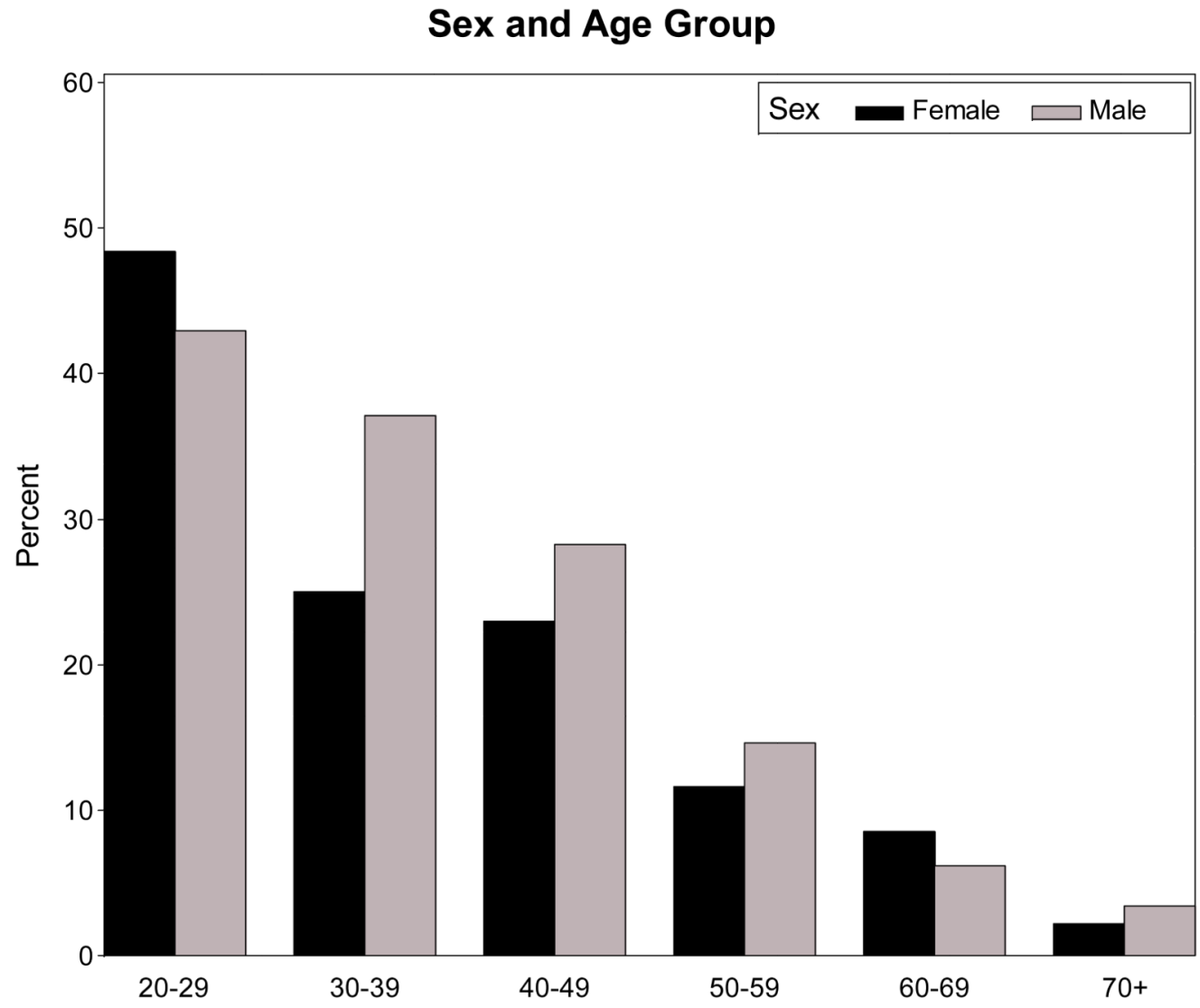

Figure 1. Proportion with ACL Reconstruction Among those with Injury by Sex and Age Group Each bar represents the proportion of the cohort, stratified by sex (dark bars for females, light bars for males) and age group (increasing from left to right) that underwent ACL reconstruction. The p-value for the interaction effect is 0.62 . 


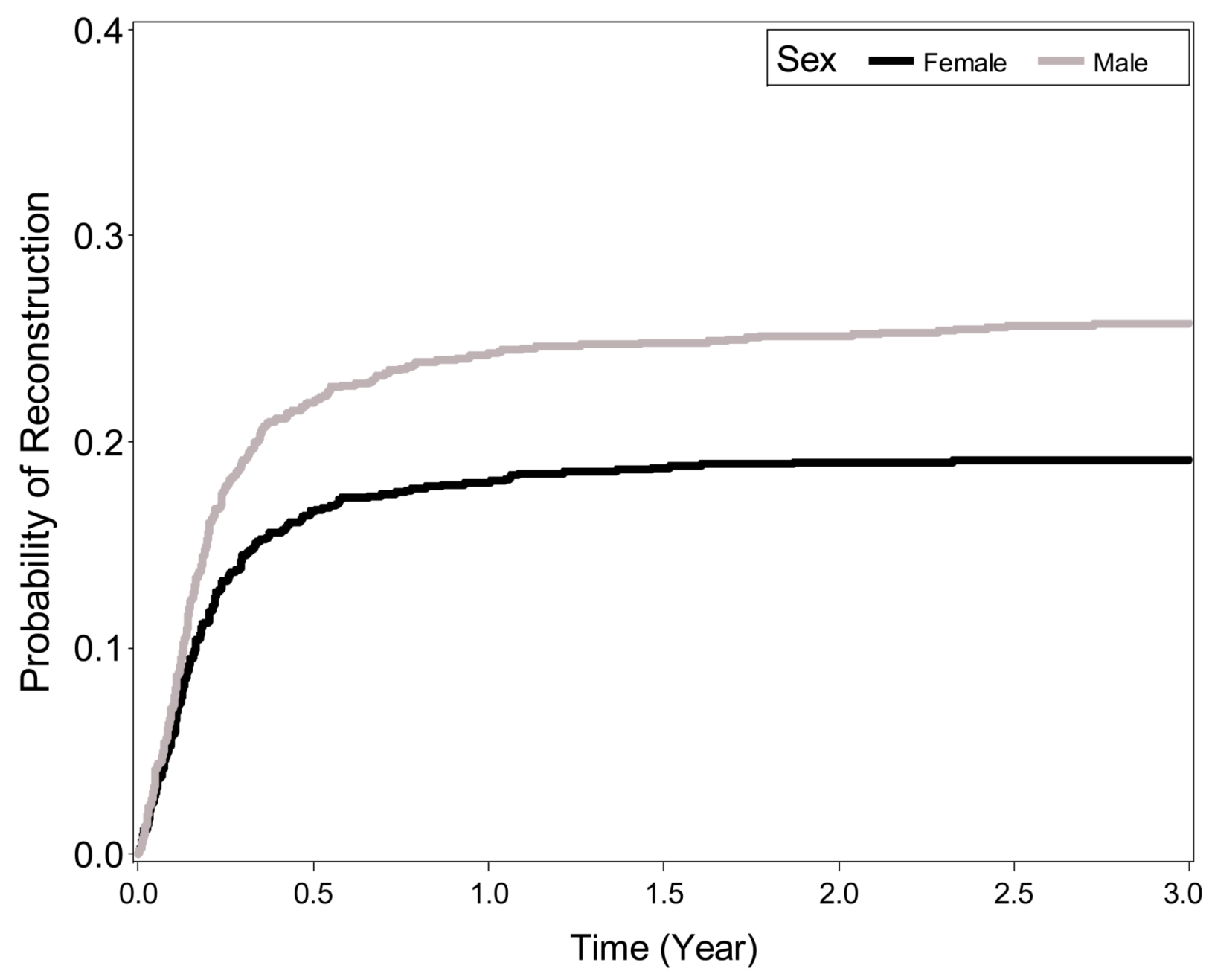

Figure 2. Kaplan-Meier Curves Depicting Probability of ACL Reconstruction for Patients with ACL Injury Stratified by Sex

Each line represents the probability of undergoing reconstruction by time since first documentation of ACL Injury stratified by sex (dark line for females, light line for males). The log-rank p-value for a difference in the survival curves is $<0.001$. 
Table 1

Characteristics of Patients with Diagnosis of ACL Injury, 2001-2008 ( N = 2370 )

\begin{tabular}{|l|l|}
\hline Characteristics & \\
\hline Sex & Percent \\
\hline Male & $52.5 \%$ \\
\hline Female & $47.5 \%$ \\
\hline Age Group & \\
\hline $20-29$ & $9.7 \%$ \\
\hline $30-39$ & $22.2 \%$ \\
\hline $40-49$ & $27.5 \%$ \\
\hline $50-59$ & $21.5 \%$ \\
\hline $60-69$ & $12.7 \%$ \\
\hline $70+$ & $6.5 \%$ \\
\hline Race & \\
\hline Non-White & $16.7 \%$ \\
\hline White & $63.9 \%$ \\
\hline Unknown & $19.4 \%$ \\
\hline Language & \\
\hline English & $94.2 \%$ \\
\hline Spanish & $3.4 \%$ \\
\hline Other & $2.4 \%$ \\
\hline SES & \\
\hline High & $31.9 \%$ \\
\hline Medium & $33.8 \%$ \\
\hline Low & $34.3 \%$ \\
\hline Insurance & \\
\hline Private Insurance & $74.6 \%$ \\
\hline Medicare & $7.8 \%$ \\
\hline Other & $4.1 \%$ \\
\hline Free Care & $9.3 \%$ \\
\hline Self-Pay & $4.1 \%$ \\
\hline & \\
\hline & \\
\hline
\end{tabular}


Table 2

ACL Reconstruction Status by Patient Characteristics

\begin{tabular}{|c|c|c|}
\hline Characteristics & No Reconstruction & Reconstruction \\
\hline \multicolumn{3}{|l|}{ Sex } \\
\hline Male & $74.3 \%$ & $25.7 \%$ \\
\hline Female & $80.9 \%$ & $19.1 \%$ \\
\hline \multicolumn{3}{|l|}{ Age Group } \\
\hline $20-29$ & $54.7 \%$ & $45.3 \%$ \\
\hline $30-39$ & $67.9 \%$ & $32.1 \%$ \\
\hline $40-49$ & $74.1 \%$ & $25.9 \%$ \\
\hline $50-59$ & $86.9 \%$ & $13.1 \%$ \\
\hline $60-69$ & $92.5 \%$ & $7.5 \%$ \\
\hline $70+$ & $97.3 \%$ & $2.7 \%$ \\
\hline \multicolumn{3}{|l|}{ Race } \\
\hline Non-White & $83.6 \%$ & $16.4 \%$ \\
\hline White & $77.0 \%$ & $23.0 \%$ \\
\hline Unknown & $73.6 \%$ & $26.4 \%$ \\
\hline \multicolumn{3}{|l|}{ Language } \\
\hline English & $76.5 \%$ & $23.5 \%$ \\
\hline Spanish & $92.2 \%$ & $7.8 \%$ \\
\hline Other & $83.6 \%$ & $16.4 \%$ \\
\hline \multicolumn{3}{|l|}{ SES } \\
\hline High & $76.1 \%$ & $23.9 \%$ \\
\hline Medium & $75.8 \%$ & $24.2 \%$ \\
\hline Low & $80.4 \%$ & $19.6 \%$ \\
\hline \multicolumn{3}{|l|}{ Insurance } \\
\hline Private Insurance & $73.3 \%$ & $26.7 \%$ \\
\hline Medicare & $92.3 \%$ & $7.7 \%$ \\
\hline Other & $72.4 \%$ & $27.6 \%$ \\
\hline Free Care & $85.5 \%$ & $14.5 \%$ \\
\hline Self-Pay & $83.1 \%$ & $16.9 \%$ \\
\hline
\end{tabular}

